\title{
Correlation of MAb subgroups with genotype in closely related Legionella pneumophila serogroup 1 strains from a cooling tower
}

\author{
P. C. LÜCK, R. J. BIRTLES* and J. H. HELBIG
}

Institut für Medizinische Mikrobiologie und Hygiene, Universitätsklinikum, TU Dresden, Dürerstrasse 24, D-01307 Dresden, Germany and * PHLS Legionella Reference Unit, Respiratory and Systemic Infections Laboratory, Central Public Health Laboratory, 61 Colindale Avenue, London NW9 $5 H T$

\begin{abstract}
Summary. Legionella pneumophila serogroup 1 strains isolated from a cooling tower during the investigation of an outbreak of Legionnaires' disease were shown previously to be related closely or indistinguishable by hybridisation-based restriction fragment length polymorphism analysis. However, these strains could be differentiated into five different MAb subgroups by comparison of their reactivity patterns with a recognised panel of monoclonal antibodies (MAbs). Pulsed-field gel electrophoresis (PFGE) of genomic fragments obtained after cleavage with rare-cutting restriction endonucleases also differentiated these strains. Four different restriction patterns were obtained with SfiI, EagI and SmaI, three restriction patterns with NotI. ApaI and SacII, and two patterns with NaeI. Generally, the restriction patterns were related closely, differing in only one or two bands. The combined results of the restriction endonuclease digestions allowed the strains to be differentiated into groups that correlated to the MAb subgroups. Both PFGE patterns and MAb subgroups were found to be stable markers. The findings demonstrated that the MAb variability seen amongst the $L$. pneumophila serogroup 1 strains from this cooling tower was not solely phenotypic.
\end{abstract}

\section{Introduction}

Legionella pneumophila serogroup 1 is the most frequent cause of Legionnaires" disease, and also the member of the Legionellaceae family isolated most frequently from environmental sources. ${ }^{1}$ The ubiquity of legionellae in natural and artificial aquatic environments complicates the establishment of an epidemiological link between isolates from the environment and those from patients. Several techniques. including monoclonal antibody (MAb) subgrouping. ${ }^{2}$ electrophoretic alloenzyme typing. ${ }^{6}$ plasmid analysis ${ }^{1.7}$ and analysis by pulsed-field gel electrophoresis (PFGE) ${ }^{1.8 .9}$ have been described for the subtyping of L. pneumophila serogroup 1 .

Harrison et al..$^{7}$ reported the isolation of a number of L. pneumophila serogroup 1 strains from the same environmental source. Although these strains were indistinguishable by a genotypic typing method, based on comparison of restriction fragment length polymorphism (RFLP) patterns obtained after hybridisation with cloned DNA fragment probes, they could be differentiated phenotypically into different $M A b$

Received 22 Sept. 1994: revised version accepted 9 Jan. 1995. subgroups. Harrison et al..$^{7}$ thus concluded that phenotypic variation of a single parent strain had occurred within the environmental site. Similar results were reported later by Struelens et $a l^{4}{ }^{4}$ during an investigation of a nosocomial outbreak of Legionnaires' disease.

This report presents evidence that PFGE analysis can be used successfully for subtyping the $L$. pneumophila serogroup 1 strains isolated by Harrison et al.' which were indistinguishable by hybridisationbased RFLP analysis. The PFGE groups obtained are compared with the MAb subgroups for the same set of strains.

\section{Materials and methods}

\section{Bacterial strains}

The nine L. pneumophila serogroup 1 strains used in this investigation are listed in the table. All these strains were isolated from a cooling tower in London.?

\section{RFLP typing}

The strains listed in the table were typed by probing Ncil-restricted chromosomal DNA with cloned EcoRI 
Table. Correlation of MAb phenotype and genotype in closely related L. pneumophila serogroup 1 strains isolated from a cooling tower in London

\begin{tabular}{|c|c|c|c|c|c|c|c|c|c|c|c|}
\hline \multirow{2}{*}{$\begin{array}{l}\text { Strain } \\
\text { no. }\end{array}$} & \multirow{2}{*}{$\begin{array}{l}\text { LRU* } \\
\text { no. }\end{array}$} & \multirow{2}{*}{$\begin{array}{l}\text { MAb } \\
\text { type }\end{array}$} & \multirow{2}{*}{$\begin{array}{l}\text { PFGE } \\
\text { type }\end{array}$} & \multicolumn{7}{|c|}{$\begin{array}{l}\text { Restriction pattern type obtained } \\
\text { with enzymes }\end{array}$} & \multirow{2}{*}{$\begin{array}{l}\text { RFLP } \\
\text { pattern }\end{array}$} \\
\hline & & & & $S f i \mathrm{I}$ & NotI & SmaI & ApaI & EagI & NaeI & SacII & \\
\hline 1 & LC751a & Bellingham & A & 1 & 1 & 1 & 1 & 1 & 1 & 1 & 47 \\
\hline 2 & $\mathrm{LC} 751 \mathrm{~b}$ & Philadelphia & B & 2 & 2 & 2 & 2 & 2 & 2 & 2 & 1 \\
\hline 3 & LC751c & Benidorm & $\mathrm{C}$ & 3 & 3 & 3 & 3 & 3 & 2 & 3 & 1 \\
\hline 4 & LC751e & OLDA & $\mathrm{D}$ & 2 & 2 & 4 & 2 & 2 & 2 & 2 & 1 \\
\hline 5 & LC751f & Oxford & $\mathrm{E}$ & 4 & 3 & 1 & 2 & 4 & 2 & 2 & 1 \\
\hline 6 & $\mathrm{LC} 751 \mathrm{j}$ & Benidorm & $\mathrm{C}$ & 3 & 3 & 3 & 3 & 3 & 2 & 3 & 1 \\
\hline 7 & LC752f & OLDA & D & 2 & 2 & 4 & 2 & 2 & 2 & 2 & 1 \\
\hline 8 & LC752y & Oxford & $E$ & 4 & 3 & 1 & 2 & 4 & 2 & 2 & 1 \\
\hline 9 & LC752o & Oxford & $\mathrm{E}$ & 4 & 3 & 1 & 2 & 4 & 2 & 2 & 1 \\
\hline
\end{tabular}

*PHLS Legionella Reference Unit, Central Public Health Laboratory, Colindale, London.

fragments (probes $\lambda$ NS20 and $\lambda$ NS21) derived from $L$. pneumophila strain Knoxville-1. ${ }^{10}$

\section{MAb subgrouping}

MAb subgrouping was performed with the MAbs of the standardised subgrouping scheme. ${ }^{2}$ These MAbs were either prepared in our laboratory, obtained from m-tech, Atlanta, GA, USA (MAb 3), or kindly provided by Jean Joly, University of Quebec, Canada (MAb 5 and MAb 7). ${ }^{5}$ The procedure used for MAb subgrouping was the indirect immunofluorescence test, as described previously. ${ }^{5}$

\section{PFGE analysis of genomic DNA}

Intact genomic DNA was prepared ${ }^{11}$ from $L$. pneumophila serogroup 1 strains and restricted with SfiI, NotI, SmaI, ApaI, EagI NaeI or SacII (New England Biolabs). Cleavage was for $15 \mathrm{~h}$ at $50^{\circ} \mathrm{C}$ for $S f i \mathrm{I}$, at $25^{\circ} \mathrm{C}$ for $S m a \mathrm{I}$, and at $37^{\circ} \mathrm{C}$ for $N o t \mathrm{I}, A p a \mathrm{I}$, EagI, SacII and NaeI, according to the manufacturer's instructions. The plugs containing cleaved DNA were loaded on agarose (GTG-agarose; Biometra, Göttingen, Germany) $1 \%$ w/v in Tris-borate-EDTA buffer (44.5 mM Tris, $44.5 \mathrm{~mm}$ boric acid, $1 \mathrm{~mm}$ EDTA, $\mathrm{pH} 8 \cdot 3$ ) gels for $S$ fi and $N o t \mathrm{I}$, or on agarose $1.3 \% \mathrm{w} / \mathrm{v}$ gels for the remaining enzymes. PFGE was performed with Rotaphor equipment (Biometra) at $13^{\circ} \mathrm{C}$. Runs were carried out with the following parameters: for $S f i \mathrm{I}$, electrophoresis for $34 \mathrm{~h}$ with pulse times of $60-5 \mathrm{~s}$, angles of $100-125^{\circ}$ and a voltage of $200-180 \mathrm{v}$, all with logarithmic ramping; for Not $\mathrm{I}$, electrophoresis for $34 \mathrm{~h}$ with pulse times of $100-5 \mathrm{~s}$, angles of $100-125^{\circ}$ and a voltage of $200-180 \mathrm{v}$, all with logarithmic ramping; and for the other enzymes, electrophoresis for $30 \mathrm{~h}$ with pulse times of 3-2 s, angles of $120-130^{\circ}$ and a voltage of $180-140 \mathrm{v}$, all with linear ramping. Yeast chromosomes (Saccharomyces cerevisiae WAY 5-4A) and Lambda concatemers (Biometra) were used as DNA size markers. PFGE gels were stained with ethidium bromide, photographed under UV light, and subjected to visual analysis. Different patterns were assigned the numbers 1-4. Eclectic PFGE types, determined by the combination of data obtained from individual PFGE patterns, were assigned the letters A-E.

\section{Results}

The results of the hybridisation-based RFLP analysis of the strains used in this study have been published previously. ${ }^{7}$ Strains belonging to MAb subgroups Philadelphia, Benidorm, OLDA and Oxford were

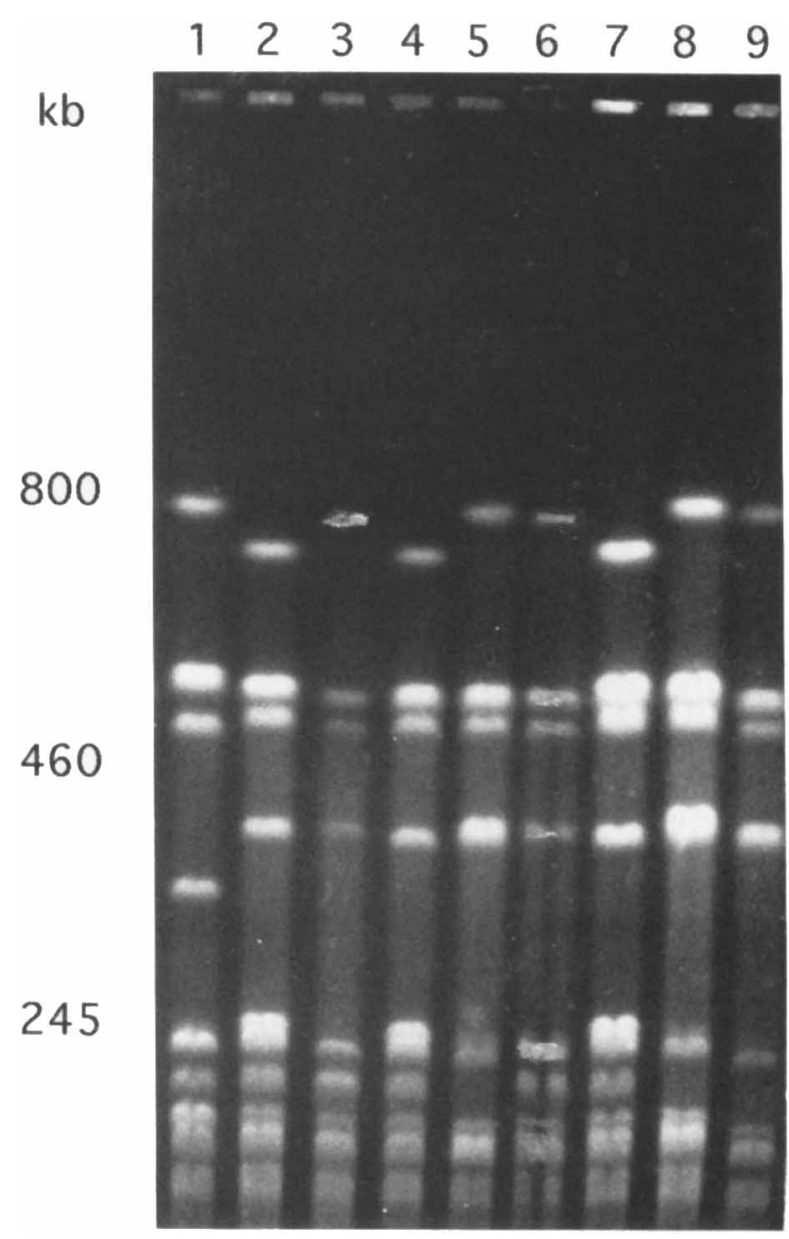

Fig. 1. PFGE of Sfil-cleaved DNA of L. pneumophila serogroup 1 strains. Lane numbers correspond to the strain numbers listed in the table. 


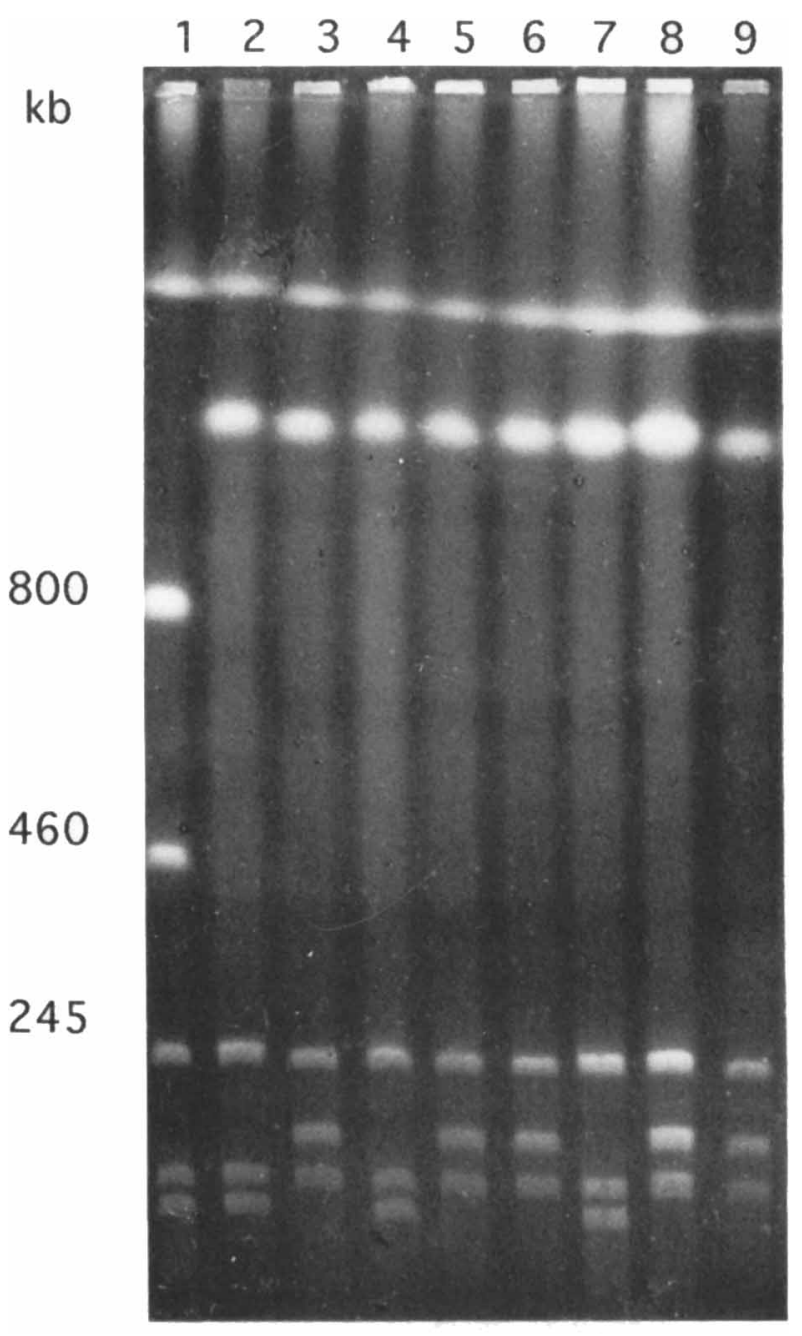

Fig. 2. PFGE of Notl-cleaved DNA of L. pneumophila serogroup 1 strains. Lane numbers correspond to the strain numbers listed in the table.

indistinguishable (RFLP pattern 1). The strain belonging to the Bellingham MAb subgroup produced a similar, but distinguishable RFLP pattern (RFLP pattern 47), differing in only one band from that of the other strains.

The results of the MAb subgrouping and the PFGE analysis, based on the SfiI, NotI, ApaI, SacII, SmaI, EagI and NaeI profiles of the genomic DNA of the nine strains tested, are summarised in the table. Examples of the restriction patterns of the strains are given in figs. 1 and 2. Enzymes Sfil, SmaI and EagI produced four restriction patterns; Not I, ApaI and SacII produced three patterns; and NaeI produced two patterns. Digestion with SfiI and EagI produced indistinguishable restriction patterns for strains belonging to the Philadelphia and the OLDA subgroups. Strains belonging to the Bellingham and Oxford subgroups were indistinguishable when their Smal cleavage patterns were compared. PFGE analysis of the strain belonging to the Bellingham subgroup produced restriction patterns that differed most clearly from those of the strains belonging to the other four $\mathrm{MAb}$ subgroups. PFGE analysis with Nael differentiated the strains into two groups corresponding to their RFLP patterns. The eclectic PFGE groups A, B,
$\mathrm{C}, \mathrm{D}$ and $\mathrm{E}$ corresponded to the MAb subgroups Bellingham, Philadelphia, Benidorm, OLDA and Oxford, respectively. All strains of the same $\mathrm{MAb}$ subgroup displayed identical PFGE restriction patterns whichever restriction enzyme was employed. The reproducibility of the NotI and SfII restriction patterns obtained for each strain was demonstrated by achieving identical results on two separate occasions.

\section{Discussion}

The thorough investigation of outbreaks or single cases of nosocomial or community-acquired Legionnaires' disease requires a combination of epidemiological and microbiological data. In order to identify a given environmental reservoir (e.g., hot water supply system, cooling tower, evaporative condenser, whirlpool spa or decorative fountain) as the source of infection, a comparison of the phenotypic and genotypic characteristics of clinical isolates with isolates obtained from that environmental source is required. The ability of a specific technique to differentiate unrelated strains into different groups is integral to the usefulness of a technique in outbreak investigation. Thus, the differentiating power of $\mathrm{MAb}$ subgrouping can be considered limited as only 10 subgroups have been defined. ${ }^{2.10}$ In contrast, RFLP analysis $^{i .10}$ and electrophoretic alloenzyme typing ${ }^{6}$ have been reported to differentiate $L$. pneumophila serogroup 1 strains into 47 and 62 subtypes, respectively. However, MAb subgrouping has the advantage that it is easy and quick to perform, and can be used directly in antigen detection assays of patient specimens. ${ }^{12,13}$ Such assays are of great benefit when the causative agent cannot be isolated by culture.

The usefulness of MAb subtyping has been questioned in several studies in which genotypically homologous L. pneumophila serogroup 1 strains, isolated from the same environmental source, expressed different MAb phenotypes (MAb reactivity patterns). ${ }^{4,7}$ These studies assumed that MAb phenotypic variation occurred amongst genetically homogeneous strains. ${ }^{4,7}$ To investigate this assumption further, PFGE analysis was applied to the subtyping of L. pneumophila serogroup 1 strains isolated originally from the same cooling tower in London, since PFGE analysis has been used previously for the successful subtyping of $L$. pneumophila serogroups 1 and $6 .^{3.8 .9}$

All strains used in this study displayed very similar PFGE types, with differences in only one or two bands (figs. 1 and 2), supporting the suggestion of Harrison et $a l^{7}$ that these strains were derived originally from a single parent strain. Although none of the seven restriction endonucleases used could differentiate the strains-in which there were representatives of five MAb subgroups - into more than four different PFGE subtypes, a strong correlation between MAb subgroup and PFGE subtype was found. This link suggests that the observed variation in MAb phenotype was ac- 
companied by minor changes in the genome structure, and, therefore, that MAb reactivity may be a useful indicator of diverging genotypic lines amongst genotypically closely related strains. As all strains of a given $\mathrm{MAb}$ subgroup showed indistinguishable restriction patterns with each of the restriction endonucleases applied, spontaneous mutations leading to the alteration of restriction sites within the genome of the strains during subculturing could be excluded. The observation that similar PFGE types were found with different restriction endonucleases also supports this conclusion. Thus $S f \mathrm{II}$ and $E a g \mathrm{I}$, despite recognising different restriction sites, both differentiated the strains into four PFGE subtypes, each subtype comprising the same strains with both enzymes. PFGE anaiysis also indicted that the genomic structure of these $L$. pneumophila strains was stable over multiple passages on artificial media, concurring with previous findings from RFLP analysis ${ }^{10}$ and PFGE analysis. ${ }^{14}$ Furthermore, technical variations during preparation of DNA and during electrophoresis did not influence the restriction patterns significantly as restriction with NotI and SfiI yielded identical profiles on two separate occasions.

The MAbs used in the standardised subgrouping scheme $^{2}$ recognise major lipopolysaccharide epitopes on the surface of $L$. pneumophila serogroup 1 strains. The stability of these epitopes, as determined by their reactivity with the MAbs, has been debated by various groups. Several workers have reported stability of the $M A b$ reactivity of strains, even after multiple passages on either BCYE agar, ${ }^{2,15}$ at various temperatures, ${ }^{16}$ or

\section{References}

1. WHO. Epidemiology, prevention and control of legionellosis: memorandum from a WHO meeting. Bull WHO 1990; 68: $155-164$.

2. Joly JR, McKinney RM, Tobin JO, Bibb WF, Watkins ID, Ramsay D. Development of a standardized subgrouping scheme for Legionella pneumophila serogroup 1 using monoclonal antibodies. $J$ Clin Microbiol 1986; 23: $768-771$.

3. Hlady WG, Mullen RC, Mintz CS, Shelton BG, Hopkins RS, Daikos GL. Outbreak of Legionnaires' disease linked to a decorative fountain by molecular epidemiology. $A m J$ Epidemiol 1993; 138: 555-562.

4. Struelens MJ, Maes N, Rost F et al. Genotypic and phenotypic methods for the investigation of a nosocomial Legionella pneumophila outbreak and efficacy of control measures. $J$ Infect Dis 1992; 166: 22-30.

5. Lück PC, Helbig JH, Ehret W, Marre R, Witzleb W. Subtyping of Legionella pneumophila serogroup 1 strains isolated in Germany using monoclonal antibodies. Int $J$ Med Microbiol Virol Parasitol Infect Dis 1992; 277 : 179-187.

6. Selander RK, McKinney RM, Whittam TS et al. Genetic structure of populations of Legionella pneumophila. $J$ Bacteriol 1985; 163: 1021-1037.

7. Harrison TG, Saunders NA, Haththotuwa A, Hallas G, Birtles RJ, Taylor AG. Phenotypic variation amongst genotypically homogeneous Legionella pneumophila serogroup 1 isolates: implications for the investigations of outbreaks of Legionnaires' disease. Epidemiol Infect 1990; 104: $171-180$.

8. Ott M, Bender L, Marre R, Hacker J. Pulsed field electrophoresis of genomic restriction fragments for the detection of nosocomial Legionella pneumophila in hospital water supplies. J Clin Microbiol 1991; 29: 813-815. in cultured Acanthamoeba cells (personal unpublished results). In contrast, Rodgers et al. ${ }^{17}$ and Mintz and $\mathrm{Zou}^{18}$ reported changes in the MAb reactivity patterns of strains during in-vitro cultivation. Neither of these two latter groups reported whether the observed $\mathrm{MAb}$ phenotypic variations were accompanied by genotypic change. Thus, the question of whether legionellae change the epitopes of their surface antigen composition in vivo remains unclear. The results of the present study argue for the co-existence of genotypically distinguishable-albeit closely relatedstrains of L. pneumophila serogroup 1 in a specific environmental reservoir, rather than genotypically homologous organisms exhibiting phenotypic variation.

In keeping with the findings of other groups, ${ }^{4,9}$ PFGE analysis was found to detect genotypic differences in $L$. pneumophila serogroup 1 strains indistinguishable by hybridisation-based RFLP analysis. No evidence of MAb phenotypic variation amongst genotypically homologous strains was found, and it was concluded that MAb subgrouping can still be considered a useful and stable tool for the differentiation of $L$. pneumophila serogroup 1 strains. However, it is clear that a combined use of genotypic and MAb typing methods in epidemiological studies relating to cases of Legionnaires' disease remains preferable.

We thank Jutta Müller, Sigrid Gäbler and Sylvia Petsche for technical assistance and Volker Bellmann for preparing the photographs. This study was supported by the Deutsche Forschungsgemeinschaft (Lu 485/1-1).

9. Schoonmaker D, Heimberger T, Birkhead G. Comparison of ribotyping and restriction enzyme analysis using pulsedfield gel electrophoresis for distinguishing Legionella pneumophila isolates obtained during a nosocomial outbreak. J Clin Microbiol 1992; 30: 1491-1498.

10. Saunders NA, Harrison TG, Haththotuwa A, Kachwalla N, Taylor AG. A method for typing strains of Legionella pneumophila serogroup 1 by analysis of restriction fragment length polymorphisms. $J$ Med Microbiol 1990; 31 : 45-55.

11. Lück PC, Bender L, Ott M, Helbig JM, Hacker J. Analysis of Legionella pneumophila serogroup 6 strains isolated from a hospital warm water supply over a three-year period by using genomic long-range mapping techniques and monoclonal antibodies. Appl Environ Microbiol 1991; 57: 3226-3231.

12. Brown SL, Bibb WF, McKinney RM. Use of monoclonal antibodies in an epidemiological marker system: a retrospective study of lung specimens from the 1976 outbreak of Legionnaires' disease in Philadelphia by indirect fluorescent-antibody and enzyme-linked immunosorbent assay methods. J Clin Microbiol 1985; 21 : 15-19.

13. Mahoney FJ, Hoge CW, Farley TA et al. Communitywide outbreak of Legionnaires' disease associated with a grocery store mist machine. J Infect Dis 1992; 165: 736-739.

14. Bender L, Ott M, Marre R, Hacker J. Genome analysis of Legionella spp. by orthogonal field alteration gel electrophoresis (OFAGE). FEMS Microbiol Lett 1990; 72: 253-258.

15. Joly JR, Winn WC. Correlation of subtypes of Legionella pneumophila defined by monoclonal antibodies with epidemiological classification of cases and environmental sources. J Infect Dis 1984; 150: 667-671.

16. Edelstein PH, Beer KB. DeBoynton ED. Influence of growth temperature on virulence of Legionella pneumophila. Infect Immun 1987; 55: 2701-2705. 
17. Rodgers JE, Eisenstein BI, Engleberg NC. Spontaneous changes in lipopolysacharide and monoclonal antibody binding in a single line of Legionella pneumophila serogroup 1 cells. In: Barbaree JM, Breiman RF, Dufour AP (eds) Legionella-current status and emerging perspectives. Washington, DC, American Society for Microbiology. 1993: 73-74.
18. Mintz, CS, Chang Hua Zou. Isolation of a Legionella pneumophila mutant that produces antigenically altered lipopolysacharide. In: Barbaree JM, Breiman RF, Dufour AP (eds) Legionella-current status and emerging perspectives. Washington, DC, American Society for Microbiology. 1993: 83-85. 